\title{
Evaluating the ALOCOM Approach for Scalable Content Repurposing
}

\author{
Katrien Verbert ${ }^{1}$ and Erik Duval ${ }^{1}$ \\ ${ }^{1}$ Dept. Computerwetenschappen, Katholieke Universiteit Leuven, \\ Celestijnenlaan 200A, B-3001 Leuven, Belgium \\ \{Katrien.Verbert,Erik.Duval\}@cs.kuleuven.be
}

\begin{abstract}
In this paper, an evaluation is presented of a framework that supports flexible content repurposing. Unlike the usual practice where content components, such as slides, images, definitions, text fragments, tables, or diagrams, are assembled manually through copy and paste, the framework enables on-the-fly access and repurposing. Retrieval of relevant components is enabled by automatic decomposition of legacy content and storage of individual components, enriched with metadata. Furthermore, the automatic assembly of these components in standard authoring tools is supported. The evaluation presented in this paper aims to assess the effectiveness and efficiency of such content reuse for presentations.
\end{abstract}

Keywords: content models, repurposing, metadata, repositories, evaluations

\section{Introduction}

Learning objects (LOs) and their reusability are important current research topics within the learning technology community. In various publications, it is argued that reuse not only saves time and money [4][19], but also enhances the quality of digital learning experiences, resulting in efficient, economic and effective learning [5].

There is an inverse relationship between the size of a LO and its reusability [28]. As the LO's size decreases (lower granularity), its potential for reuse increases. Many shared LOs are, however, coarse-grained compositions and as such difficult to repurpose [12]. Paragraphs, images or diagrams are frequently assembled manually by copy-paste actions. However, it is possible to repurpose LOs effectively, if their components can be accessed on-the-fly. This requires innovative and flexible LO modeling [5].

In earlier work, we developed an abstract learning object content model (ALOCOM) that is a framework for LOs and their components [7]. The model defines both LO component types and relationships between components. As such, the model enables structuring of composite LOs and is a solid basis for the proposed dynamic approach. In [26], we presented a framework that supports the approach for presentations. The motivation for choosing presentations was based on their extensive use [8] and the fact that slides are often designed as self-contained pieces of content, representing a single topic or idea. The framework transforms (legacy) content into a 
representation compliant with the ALOCOM model. In this transformation process, content is decomposed and components are individually stored in a LO repository, enriched with metadata. For scalability purposes, duplicate detection techniques are used to detect reuse for different component types in order to avoid duplicates. Furthermore, a ranking function is included that assigns a comparative value to a component based on its reuse, enabling ordering of result lists.

The fine-grained components stored in the repository are the necessary building blocks for supporting flexible content reuse. This requires a tight integration into standard authoring tools, as authors prefer to use authoring tools they are familiar with to create content. We developed a plug-in for Microsoft PowerPoint that enables users to search components, such as images, definitions, slides, text fragments, diagrams or tables, from within the application.

In this paper, we present an evaluation of the approach for presentation repurposing. A user evaluation has been performed that assessed the usability and utility of the plug-in for Microsoft PowerPoint. The goals of the evaluation were threefold: (i) to assess the efficiency and effectiveness of the approach for repurposing presentations; (ii) to assess the subjective acceptance of the ALOCOM interface; (iii) to determine to which level of granularity decomposition is relevant. A follow-up evaluation was necessary to confirm the results and assessed the quality of the created presentations.

In the next section, we briefly outline the ALOCOM architecture. The user evaluation and the quality evaluation are presented in section 3 and 4 , followed by a discussion in section 5. Related work is presented in section 6. Conclusions and remarks on future work conclude this paper.

\section{The ALOCOM Architecture}

In the ALOCOM architecture, the server relies on the ARIADNE Knowledge Pool System [24] for storage of content components and their metadata. The architecture is depicted in Fig. 1 and consists of the following components:

- Client side applications that enable content uploading to and component retrieval from the repository from within authoring tools. We developed a plug-in that provides these functionalities for Microsoft PowerPoint.

- The Disaggregation module supports the actual decomposition. In the case of presentations, the presentation is decomposed into slides, and each slide is further decomposed into images, tables, diagrams, definitions and text fragments. The current implementation of this module supports the approach for PowerPoint presentations. Components are extracted, preview thumbnails are generated and results are stored through the AdvancedContentInserter.

- The AdvancedContentInserter provides support for storing not only complete LOs, but also components that are contained in the LO, for instance components stored in a SCORM content package or components that were extracted by the Disaggregation module. Reuse is detected using simple metrics that compute similarities between incoming and stored components, such as the cosine similarity measure for detecting overlaps between text fragments and hash 
functions for detecting identical images [20]. In the next step, LOM metadata is generated for each component using the Automatic Metadata Generation framework (AMG) [1]. Relationship metadata are added that describe different relationships between parent and child components ("isPartOf", "hasPart") and between components ("ordering"). Finally, LO components are stored in the ALOCOM repository using the ARIADNE insert service [24].

- The ranking module assigns comparative values to components based on their reuse that enables ordering of result lists when a user searches for relevant objects, placing components with a high probability at the top of the list [16].
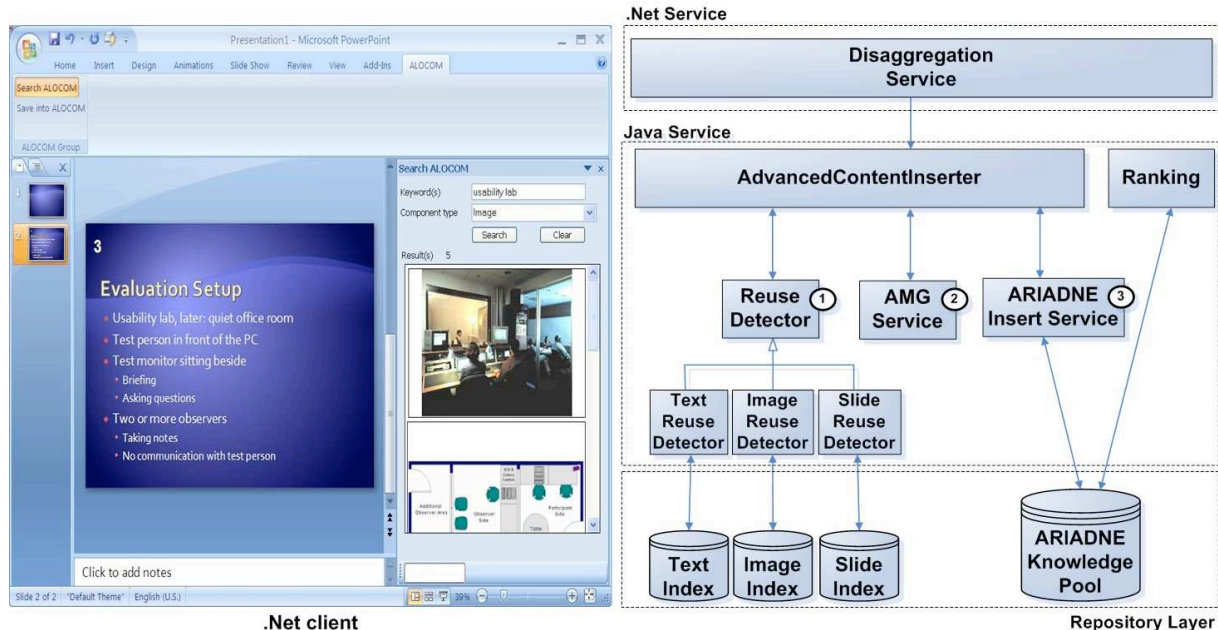

Fig. 1. The ALOCOM plug-in for PowerPoint (left) The ALOCOM Architecture (right)

The ALOCOM repository is currently filled with 62841 components that were extracted from 814 presentations. These components include 18149 slides, 7028 images, 226 tables, 30 diagrams and 35460 text fragments. We developed a plug-in for Microsoft PowerPoint that enables authors to automatically repurpose these components from within the application. As shown in Fig. 1, a custom Office Task Pane (on the right side) is used for integrating this functionality in Microsoft PowerPoint. This is accomplished with Visual Studio 2005 Tools for the Microsoft Office System [27].

The plug-in enables authors to search the repository for components they wish to repurpose in the presentation they are editing. An author can specify the component type, such as reference, definition, example, slide, image, or text fragment, and descriptive keywords. Thumbnails of components that satisfy the search criteria are shown in the Task Pane and the author can incorporate them into the current presentation by a single mouse-click. Metadata associated with the component is shown if the author hovers the mouse pointer over a component in the result list. Authors can add presentations to the repository by clicking the "Save into ALOCOM" button that we added to the standard PowerPoint menu. 


\section{User Evaluation}

The user evaluation assessed the usability and utility of the ALOCOM plug-in for Microsoft PowerPoint. The goals of the evaluation were the following:

- to assess the efficiency and effectiveness of the approach for repurposing presentation components;

- to assess the subjective acceptance of the ALOCOM interface;

- to determine to which level of granularity decomposing presentations is useful.

\subsection{Study Description}

The study was conducted in October 2006 at K.U. Leuven. Each session involved one participant, who performed two tasks during a single session. There were 20 participants in the study, which typically results in a reasonably tight confidence interval [15]. Participants were mainly members of the junior staff of the Computer Science Department at K.U. Leuven.

Tasks. Each participant was asked to create two presentations: one on inheritance and one on exceptions in the programming language Java. The participants were divided in two groups. The first group created the presentation on exceptions in Java without ALOCOM support, and the presentation on inheritance in Java with ALOCOM support. They could use all information available on the World Wide Web for both presentations. The second group did the same, but in a different order. This group created the presentation on inheritance in Java without ALOCOM support, and the presentation on exceptions in Java with ALOCOM support.

We refer to the presentation created without ALOCOM support as without-alocom presentation and the presentation created with ALOCOM support as with-alocom presentation in the remainder of this paper.

78 presentations on both topics were gathered by a Google-search and uploaded to the repository: as described above, they were automatically decomposed and the components were automatically described. In total, 10281 components were made available for reuse, including 2964 slides, 933 images, 6367 text fragments, 12 tables and 5 diagrams.

Data Collection. Camtasia Studio $^{1}$ was used to record participant interactions, capturing the screen, voice and webcam video. Participants were also asked to complete a questionnaire after the tasks. The questionnaire was adopted from a usability evaluation of the ARIADNE search tool [13].

Measurements. The following characteristics were measured for the experiment:

- Time-on-task: represents the time needed to finish each task. The aim of the time comparison is to investigate whether the use of the ALOCOM plug-in can lead to savings in time. We are aware that time is influenced by other factors; however,

\footnotetext{
${ }^{1}$ http://www.techsmith.com/camtasia.asp
} 
we included this comparison in order to obtain a first indication of improvements for time-on-task.

- Manual versus semi-automatic reuse: The distinction is made between manually reused components and semi-automatically reused components. Manually reused components are components that were added to the presentation by copy-pasting or reproducing existing content, typically found through Google. Semiautomatically reused components are those components that were found and inserted using the ALOCOM plug-in. By measuring and comparing both types of content reuse, we obtain a success rate indication of the ALOCOM approach for repurposing content, as authors typically tried the semi-automatic approach first and inserted content manually if no relevant components were found through the ALOCOM plug-in.

- Component granularity: the granularity of semi-automatically reused component types is measured in order to determine to which level of granularity decomposition of presentations is relevant.

- Satisfaction: user satisfaction was assessed through a questionnaire filled in by each participant after finishing the tasks. Questionnaire questions intended to measure the overall satisfaction on the usage of the plug-in.

\subsection{Results}

Time. Table 1 shows the average time participants spent on creating without-alocom and with-alocom presentations. At first sight, the difference is relatively limited: on average, 20.03 minutes were spent creating the without-alocom presentation and 17.79 minutes creating the with-alocom presentation. However, not all participants created presentations similar in length, covered sub-topics or quality in general.

Size normalizations were applied that were adopted from the software quality field [6]. A simple normalization that takes into account the number of slides in the presentation shows that on average 3.32 minutes were spent per slide in a withoutalocom presentation, whereas 2.2 minutes were spent per slide created with ALOCOM support.

A second normalization was applied that takes into account the number of subtopics. Some participants created presentations covering many sub-topics, such as polymorphism and dynamic binding for the presentation on inheritance, while others provided only a definition and an example. If we consider the number of sub-topics, we see that on average 4.5 minutes were spent on a sub-topic in a without-alocom presentation and 2.9 minutes on a with-alocom presentation sub-topic.

To statistically establish whether the difference between these average values is real or a by-product of natural variance, we applied a Paired-Samples T Test. The null hypothesis is that there is no difference between the required creation time for withalocom and without-alocom presentations. Our alternative hypothesis is that there is indeed a difference. Results were obtained with a normal distribution.

We can reject the null hypothesis for normalized time values. Thus, taking into account the size of presentations, significant time savings are realized when creating presentations with support to automatically repurpose existing presentation components. To validate these results, a second evaluation has been performed that 
assessed the quality of the created presentations. This evaluation is presented in section 4.

Table 1. Time (in minutes)

\begin{tabular}{|l|c|c|c|}
\hline & $\begin{array}{l}\text { without-alocom } \\
\text { presentation }\end{array}$ & with-alocom & $\begin{array}{l}\text { Sig.(2- } \\
\text { tailed) }\end{array}$ \\
\hline Total time & 20.03 & 17.79 & 0.147 \\
\hline Time normalized by number of slides & 3.32 & 2.2 & 0.001 \\
\hline Time normalized by number of subtopics & 4.5 & 2.9 & 0.016 \\
\hline
\end{tabular}

Reuse in With-Alocom Presentations. With-alocom presentations were further analyzed. The distinction is made between manual reuse, semi-automatic reuse and new components. Manually reused components are components that were added to the presentation by copy-pasting or reproducing existing content, found by a web search. Semi-automatically reused components are those components that were found and inserted using the ALOCOM plug-in. New components represent content the participant created from scratch, without using an existing resource.

Fig. 2(a) shows reuse patterns of individual participants. Some participants reused about the same amount of components manually as semi-automatically. Also, the amount of new components is high for some participants (more than $40 \%$ ). Few participants created presentations without manual reuse.

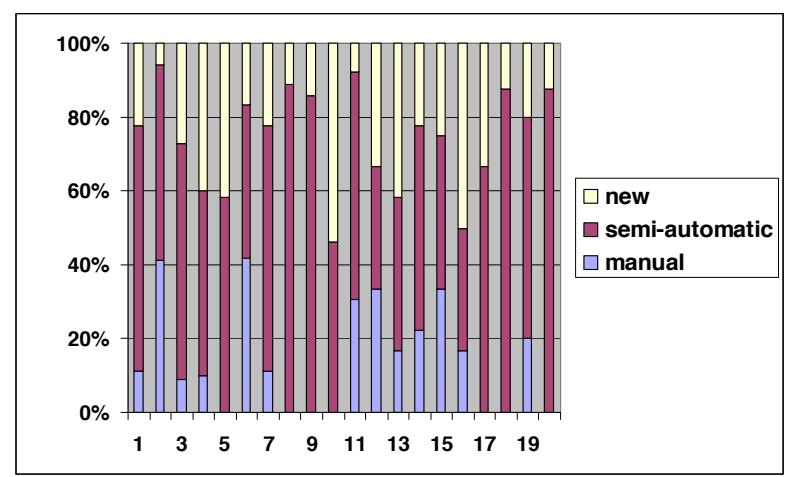

a)

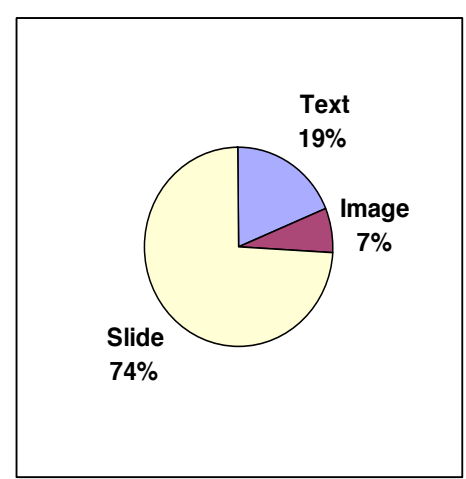

b)

Fig. 2. Reuse patterns of individual participants (a); reuse/component type (b)

Table 2 shows that on average $57 \%$ of presentation components are semiautomatically reused using the ALOCOM plug-in. $18 \%$ of the components were reused manually, whereas $25 \%$ are new components. There is no significant difference if we compare this data for the presentation on exceptions in Java and the presentation on inheritance in Java, although more components were available covering topics on inheritance. The values were normally distributed and compared with a Paired-Samples T Test. 
Comparing manual and semi-automatic reuse, we see that $76 \%$ of reused components were reused semi-automatically, whereas $24 \%$ were reused by copy-paste actions or reproduction of content. These values are a success rate indicator of the ALOCOM approach for reusing content, as participants typically tried the semi-automatic approach first and inserted content manually if no relevant components were found through the ALOCOM plug-in.

Table 2. Reuse in with-alocom presentations

\begin{tabular}{|l|l|l|l|}
\hline & Manual & Semi-automatic & New \\
\hline Overall & 0.18 & 0.57 & 0.25 \\
\hline Presentation on inheritance (1) & 0.19 & 0.58 & 0.23 \\
\hline Presentation on exceptions (2) & 0.18 & 0.55 & 0.27 \\
\hline $\begin{array}{l}\text { Comparing means (1) and (2) } \\
\text { Sig. (2-tailed) }\end{array}$ & 0.737 & 0.121 & 0.791 \\
\hline
\end{tabular}

Granularity. Fig. 2(b) shows the reuse rate for semi-automatically reused component types. Complete slides are most often reused, probably because many slides represent a single idea or topic and are thus easy to reuse in a new context. Also the reuse of text fragments is significant. This is an interesting result, as it illustrates that breaking content down to the level of a single text fragment is useful. Images were not frequently reused; however, this result is probably influenced by the topic of the presentations.

Findings and Recommendation. In this section, findings and recommendations of the participants are discussed.

Lack of Context. Some participants remarked that more context is required for successful content reuse. They want to be able to retrieve the next and previous slide for a specific slide in the result list, or even the complete presentation(s) to which the slide belonged. Similar support is needed for other component types.

Behaviour Change. It was noted that this way of reusing content requires a behaviour change, as it is different from the usual practice of copy-pasting or reproducing content. It was reported that savings in time would be remarkable; however, a period of adaptation is required.

Drag and Drop Support. Many participants expected drag and drop support for inserting components. There is click-support for inserting a component: clicking a component in the result list will insert the component at the currently selected location. However, it is not possible to drag the component to a different location in the presentation due to limitations of the PowerPoint API [22].

Garbage Content. Not all components are reusable. As components are created by decomposing existing content automatically, it was expected that not all components 
are valuable for reuse. Results are ranked according to the number of times a component is reused. Hence, the impact of this issue will decrease over time.

Less Consistent Layout. Some participants noted that it is hard to keep the layout of different components consistent. The layout of slides is automatically adapted to the template the author is using. However, if the author changed for instance the font color of a text fragment in one particular slide, this modification is preserved when reusing the slide. Although desirable in some cases, this was reported as a difficulty.

More Valuable for Reuse of Own Content. Participants remarked that the use of the ALOCOM plug-in would be most valuable for reusing their own presentations.

Overall Satisfaction. Table 3 presents the responses of participants to questions concerning the overall use of the ALOCOM plug-in. The questionnaire was adopted from a usability evaluation of the ARIADNE search tool [13]. The popular attitude scale with seven points (ranging from 1 - poor to 7 - good) was used to measure the response of participants on the overall use of the plug-in.

Table 3. Satisfaction

\begin{tabular}{|l|c|c|}
\hline & mean (ranging from 1-7) & Standard deviation \\
\hline Ease of use & 6.15 & 0.69 \\
\hline Information organization & 5.23 & 0.93 \\
\hline Use of terminology & 4.92 & 1.5 \\
\hline Navigation & 6.07 & 1.04 \\
\hline Search and reuse of components & 5.69 & 1.49 \\
\hline Result list easy to read & 4.92 & 1.5 \\
\hline
\end{tabular}

The mean for the level of ease-of-use was more than 6, meaning that the participants found the ALOCOM plug-in easy to use. The level of information organization and search and reuse of LO components was perceived as moderate (mean 5.23 and 5.69 respectively). We believe that this is related to the fact that there is a lack of context (it is not possible to automatically retrieve the original component to which a component belonged) and the fact that there is no drag and drop support.

Result lists were found rather difficult to read (mean 4.92). This result is a consequence of the fact that preview thumbnails of slides containing much content are difficult to read. We have worked on a solution that enables users to enlarge individual components. Each component in the result list has a context menu item that provides this functionality. This solution will resolve the issue if only few components are difficult to read. 


\section{Quality Evaluation}

In a follow-up evaluation, the quality of with- and without-alocom presentations was assessed by a group of 19 participants. This evaluation was necessary for obtaining a more accurate estimation of the effectiveness and efficiency of the ALOCOM approach for repurposing presentations.

Following a common practice to reduce subjectivity in a quality evaluation, an evaluation framework was used. In [9], an overview is provided of the most common dimensions of Content Quality frameworks. Four dimensions that were relevant in the context of the experiment were used to evaluate the quality of the presentations: accuracy, completeness, relevancy and conciseness.

In an accurate presentation, the content contained in the presentation is correct, reliable and free of error. Completeness is defined as the extent to which information is not missing and is of sufficient breadth and depth for the task at hand. Relevancy measures whether the content contained in the presentation is applicable and helpful for the task at hand. Finally, in a concise presentation, content is broken up into smaller chunks that can be easily shared with an audience.

Participants in the experiment were requested to read the definition of each parameter before grading the presentations. The definitions were also available during the evaluation process.

The experiment was carried out online using a web application. After logging in, the system presented users with instructions. After reading the instructions, users were presented with a list of 20 randomly selected presentations. Once users had reviewed a presentation, they were asked to give grades on a 7-point scale, from "Extremely low quality" to "Extremely high quality", for each parameter. Only participants that graded all presentations were considered in the experiment.

The experiment was available for 2 weeks. During that period, 24 participants entered the system, but only 19 completed the evaluation. From those 19 participants, 13 were postgraduate students, 1 had a Ph.D. degree and 5 were active in software development. All participants had a degree in computer science.

\subsection{Data Analysis}

Because of the inherent subjectivity in measuring quality, the first step in the analysis of the data is to estimate the reliability of the evaluation [10]. In this kind of experiment, the evaluation is considered reliable if the variability between the grades given by different reviewers to a particular presentation is significantly smaller than the variability between the average grades given to different presentations. To estimate this difference, we use the Intra-Class Correlation (ICC) coefficient [21], which is commonly used to measure the inter-rater reliability. We calculate the average ICC measure using the two-way mixed model, given that all reviewers grade the same sample of presentations. In this configuration, the ICC is equivalent to another widely used reliability measure, the Cronbachs alpha [2]. The results for each quality parameter are reported in the Table 4. 
Table 4. Intra-Class Correlation (ICC) coefficient for measuring the reliability

\begin{tabular}{|l|l|}
\hline Parameter & ICC (average, two-way mixed) \\
\hline Completeness & 0.927 \\
\hline Accuracy & 0.766 \\
\hline Conciseness & 0.881 \\
\hline Relevancy & 0.837 \\
\hline
\end{tabular}

Generally, ICC values above 0.75 indicate good reliability between measures. None of the values fall below this cut-off value. Hence, the ICC suggests that reviewers provided similar values and further statistical analysis can be performed.

The second step is to assess whether there is a difference between the average grade given to with-alocom presentations and the average grade given to withoutalocom presentations. These average values are presented in Fig. 3. To statistically establish whether the difference between average values is real or a by-product of natural variance, we applied a Paired-Samples T Test. Our null hypothesis is that there is no difference between the grades given to with-alocom and without-alocom presentations. Our alternative hypothesis is that there is indeed a difference. The results are presented in Table 5. Results were obtained with a normal distribution.

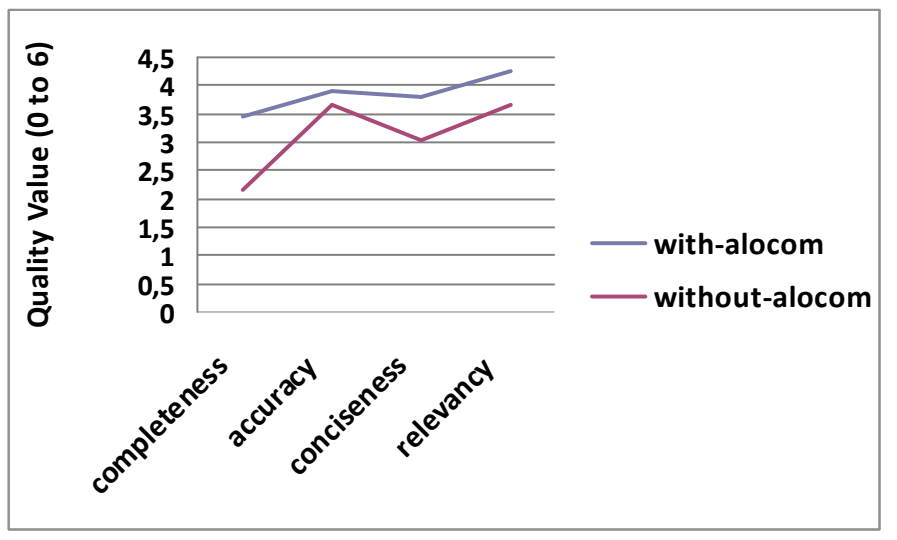

Fig. 3. Average quality grade for the different parameters

Table 5. Significance of the difference between the given grades

\begin{tabular}{|l|l|l|}
\hline Parameter & T-value & Significance (2-tailed) \\
\hline Completeness & -8.094 & 0.0 \\
\hline Accuracy & -1.412 & 0.160 \\
\hline Conciseness & -4.352 & 0.0 \\
\hline Relevancy & -2.981 & 0.003 \\
\hline
\end{tabular}


We can reject the null hypothesis for most of the parameters (completeness, conciseness and relevancy). The significant difference found in the completeness parameter indicates that users were able to create more complete presentations when provided with support to repurpose presentation components. The significant difference found in the conciseness parameter indicates that content extracted from existing presentations is more suitable for reuse as it is already presented in a form that can be shared with an audience. Furthermore, users were able to find more relevant content for with-alocom presentations. No significant difference was found in the accuracy parameter. As the presentations were created by members of the junior staff of the Computer Science Department at K.U. Leuven, it was expected that no major mistakes would be made in creating presentations on inheritance and exceptions in Java.

\section{Discussion}

Although no direct improvement in savings in time was perceived, results of the quality evaluation indicate that providing on-the-fly access to presentation components in an authoring process enhances the quality of presentations. Presentations created with ALOCOM support are significantly more complete, concise and relevant. The results in completeness are consistent with the size normalizations applied to time values. Hence, we can conclude that there is also a significant improvement in time savings.

Results of the user evaluation indicate that the plug-in can be used in a successful way: $76 \%$ of reused components were reused semi-automatically. However, usability issues need to be resolved in order to make this kind of content reuse more efficient. Most important is the context issue. The user interface should be extended with the functionality to retrieve the component to which a component in the result list originally belonged. Furthermore, it is important to enable navigation in the original structure of presentations. For instance, support is needed to retrieve the next and previous slide for a specific slide in the result list. We will integrate these functionalities in the PowerPoint plug-in.

We cannot improve the consistent layout issue, as we use built-in copy and paste functions of the PowerPoint API for adding an existent slide to a presentation. If a user would manually copy-paste a slide, the same problem with consistency arises. Drag and drop support is also difficult to integrate. We will, however, investigate possibilities to improve the way a component can be inserted.

The method we used can be classified as a "discount usability engineering" approach [14] as it is definitely not "the perfect" method for evaluation and will not give absolute results. However, it enabled us to obtain a good indication of improvements towards savings in time or enhancements of quality and to highlight usability issues. A second evaluation will be performed after resolving the aforementioned issues with a group of participants from outside the computer science field. 


\section{Related Work}

Reuse is considered to be an effective strategy for building high-quality content [25]. However, there is currently a lack of evaluation methods and metrics for measuring the impact of content reuse in terms of productivity and quality gains.

In the software engineering field, researchers have worked on metrics for measuring reuse benefits. Size, reuse rate and effort metrics are used for calculating these benefits. Furthermore, many frameworks have been presented that measure quality, in both software and information quality fields. In this paper, we have applied techniques and frameworks that are valid in our context.

In [11], an empirical evaluation is presented that assesses the impact of reuse on quality and productivity in object oriented systems. Similar to our evaluation, amount of reuse and total amount of hours spent on a system are measured. In [3], an analytical and empirical evaluation of software reuse metrics is presented. Software measures are categorized along orthogonal axes that measure attributes of the software product, such as quality of code, and attributes of the software process, such as cost of design review. In our case, the quality evaluation assessed product/content attributes, and time-on-task is an attribute of the process. Furthermore, reuse metrics that measure the amount of reuse, like our reuse measurements presented in section 4, are commonly used to estimate savings [3].

In the content management field, content reuse is reported to lead to savings in time and improvements of quality. Vasont [25] is a commercial content management system that enables organizations to create, manage and store their content for component-level reuse and delivery in multiple outputs. They report that substantial content reuse resulted in time savings in content creation, revisions, delivery, and translation. According to the study, content reuse varies by industry. Technology companies, such as software developers, have been found to achieve an average of $70 \%$ content reuse, while manufacturing companies achieve approximately $93 \%$ content reuse. Like many other commercial content management systems, the system supports reuse by manual transformation of content.

In contrast to Vasont, MagIR [8] is a system that supports automatic content transformations. Like our system, these transformations are supported for Microsoft PowerPoint presentations and include content decomposition. Decomposition is supported to the level of slides, while our system also extracts smaller components such as tables, diagrams, images and text fragments. Results of our user evaluation indicate that these fine-grained components are also often reused. MagIR is used for creation, administration and reutilization of PowerPoint slides in a corporate context and is aimed at reducing storage costs. The system has been evaluated in that context and results indicate that storage costs are significantly reduced.

Slide executive [23] is a commercial product that also supports reutilization of Microsoft PowerPoint slides. Individual slides can be retrieved in a browser. Like MagIR, decomposition is supported to the level of slides. Add-ins are provided to export PowerPoint slides to images in different formats and to import multiple images at once. However, no tight integration for component searching from within the application is provided. No information was found whether the system has been evaluated. 


\section{Conclusions}

In this paper, an evaluation was presented of our framework that supports on-the-fly repurposing of presentation components, providing an indication of the effectiveness and efficiency of such content reuse. The analysis of the results indicates that there is a significant improvement of the quality of presentations and a significant time saving benefit.

We have extended the current implementation of the framework with support for (de-)composing SCORM Content Packages and Wikipedia pages. A plug-in has been developed for Microsoft Word that enables repurposing of components from Wikipedia pages and an equivalent plug-in has been developed for the RELOAD editor [18] that supports reuse of SCORM components. Evaluations have to be performed to assess whether these related approaches result in a similar impact on effective and efficient content reuse.

Acknowledgments. We gratefully acknowledge the financial support of the K.U.Leuven research council through the BALO project, the Interdisciplinary Institute for Broadband Technology (IBBT) through the Acknowledge project, and the European Commission through the ProLearn Network of Excellence on Professional Learning [17].

\section{References}

1. Cardinaels, K., Meire, M., and Duval, E., "Automating Metadata Generation: the Simple Indexing Interface", International World Wide Web Conference, WWW 2005, May 10-14, 2005, Chiba, Japan, pp. 548-556.

2. Cronbach, L. J. (1951). "Coefficient alpha and the internal structure of tests". Psychometrika, 16, pp. 297-334.

3. Devanbu, P., Karstu, S., Melo, W. and Thomas, W. "Analytical and Empirical Evaluation of Software Reuse Metrics". In The 18th Internation Conference on Software Engineering, pages 189 -- 199, 1996. http://citeseer.ist.psu.edu/devanbu96analytical.html

4. Downes, Stephen (2001), "Learning Objects: resources for distance education worldwide", in International Review of Research in Open and Distance Learning, Vol.2, No. 1.

5. Duval, E. and Hodgins, W., "A LOM research agenda," In Proceedings of the 12th International World Wide Web Conference., Budapest, Hungary, 2003, pp. 1-9.

6. ISO/IEC 9126 -2 Software quality characteristics and metrics - External metrics (1998).

7. Jovanović, J., Gašević, D., Verbert, K., and Duval E. (2005). "Ontology of learning object content structure". In Proc. of the 12th International Conference on Artificial Intelligence in Education, Amsterdam, The Netherlands, pp. 322-329.

8. Kienreich, W., Sabol, V., Ley, T., Lindstaedt, S., Koronakis, P. and Droschl, G. (2005) "MagIR: Distributed Creation, Administration and Reutilization of Multimedia Presentation Content", in Proceedings of WM05 - Workshop IT Tools for Knowledge Management Systems, Kaiserslautern, Germany, 2005, pp. 481-486.

9. Knight, S.A. \& Burn, J.M (2005) "Developing a Framework for Assessing Information Quality on the World Wide Web”, Informing Science Journal; Vol. 8; pp. 159-172. 
10. Meire, M., Duval, E., and Ochoa, X. (2007). "SAmgI: Automatic Metadata Generation v2.0". In Proceedings of ED-MEDIA 2007, World Conference on Educational Multimedia, Hypermedia \& Telecommunications, 2007 (to appear).

11. Melo, W.L., Briand, L.C., Basili, V.R. (1995), "Measuring the Impact of Reuse on Quality and Productivity on Object-Oriented Systems", Technical Report CS-TR-3395, University of Maryland, 1995, 16 pages.

12. Motelet, O. "Enabling the Reuse of Learning Material in the Classroom", PhD Thesis http://www.dcc.uchile.cl/ omotelet/papers/Thesis_Proposal_Motelet_2004.pdf

13. Najjar, J., Klerkx, J., Vuorikari, R. and Duval, E. (2005), "Finding Appropriate Learning Objects: An Empirical Evaluation", ECDL 2005 9th European Conference on Research and Advanced Technology for Digital Libraries (Rauber, A., et al. eds. ), pp. 323-335, 2005.

14. Nielsen, J. (1989). "Usability engineering at a discount”. In Salvendy, G., and Smith, M.J. (Eds.), Designing and Using Human-Computer Interfaces and Knowledge Based Systems, Elsevier Science Publishers, Amsterdam, pp. 394-401.

15. Nielsen, J. (2006). "Quantitative Studies: How Many Users to Test?" Alertbox, June 26 2006, http://www.useit.com/alertbox/quantitative testing.html

16. Ochoa, X. and Duval, E. (2006), "Use of contextualized attention metadata for ranking and recommending learning objects", In Proceedings of the 1st international workshop on Contextualized attention metadata, Arlington, Virginia, USA, pp. 9-16.

17. ProLearn Network of Excellence on professional learning, http://www.prolearn-eu.org/

18. Reload Editor. http://www.reload.ac.uk/editor.html

19. Robson, R. "Context and the Role of Standards in Increasing the Value of Learning Objects", chapter in Online Education Using Learning Objects edited by R. McGreal, University of Athabasca, Canada.

20. Shivakumar, N. and Garcia-Molina, H. (1995), "SCAM: A Copy Detection Mechanism for Digital Documents", In Proceedings of the Second Annual Conference on the Theory and Practice of Digital Libraries, June 11-13, 1995, Austin, Texas, USA.

21. Shrout, P. E., and Fleiss, J. L. (1979). "Intraclass Correlations: Uses in Assessing Rater Reliability”. Psychological Bulletin(2), pp. 420-428.

22. Siew Moi Khor, and Art Leonard (2005), Installing and Using the Office 2003 Primary Interop Assemblies, Microsoft Office 2003 Technical Articles.

23. Slide Executive - The PowerPoint Presentation Management Library. http://www.slideexecutive.com/

24. Ternier, S. and Duval, E. "Web services for the ARIADNE knowledge pool system", in Proceedings of the 3rd Annual ARIADNE Conference (Duval, E., ed.), pp. 1-9, 2003.

25. Vasont Systems (2007) Vasont reuse case study: http://www.vasont.com/vasont/literature/contentreuse.asp

26. Verbert, K., Jovanovic, J, Gaševic, D, and Duval, E. (2005), "Repurposing Learning Object Components", In Proceedings of OTM 2005 Workshop on Ontologies, Semantics and E-learning, Agia Napa, Cyprus, pp. 1169-1178.

27. Visual Studio Tools for Office, http://msdn2.microsoft.com/en-us/library/d2tx7z6d.aspx

28. Wiley, D.A. (2003) "Learning Objects: Difficulties and Opportunities." http://wiley.ed.usu.edu/docs/lo_do.pdf 